\title{
iTRAQ-based analysis of sperm proteome from normozoospermic men achieving the rescue-ICSI pregnancy after the IVF failure
}

Xin Liu ${ }^{1 \dagger}$, Gensheng Liu ${ }^{2 \dagger}$, Juan Liu' ${ }^{1 \dagger}$, Peng Zhu ${ }^{1 \dagger}$, Jiahui Wang ${ }^{1}$, Yanwei Wang ${ }^{1}$, Wenting Wang ${ }^{1}$, Ning Li ${ }^{1}$, Xuebo Wang ${ }^{3}$, Chenglin Zhang ${ }^{1}$, Xiaofang Shen ${ }^{1,4^{*}}$ and Fujun Liu ${ }^{1 *}$

\begin{abstract}
Background: In the assisted reproduction, the infertile molecules of spermatozoa from normozoospermic men who underwent the unexplained failure of in vitro fertilization (IVF) due to the lack of sperm binding to the normal zona pellucida, and then achieved pregnancy with the rescue intracytoplasmic sperm injection (R-ICSI) remain unclear. More works are still necessary to explore this male infertile mechanism.

Methods: Normozoospermicmen with the IVF pregnancy and normozoospermic men with the R-ICSI pregnancy after the conventional IVF failure were collected. iTRAQ-based proteomic approach were performed to reveal the new infertile causes between the IVF pregnancy men and the R-ICSI pregnancy men. To validate the confidence of proteome data, the individual samples were analyzed by western blot and immunofluorescence. Further, the spontaneous acrosome reactions were measured to evaluate the sperm quality.

Results: Compared with IVF pregnancy group, 56 sperm proteins were differentially expressed in the R-ICSI pregnancy group. Bioinformatic analyses (PANTHER, DAVID, PubMed and STRING) indicated these altered sperm proteins were involved in various molecular functions: reproduction, chromosome organization, and sperm-oocyte interaction. Moreover, the confidence of proteome data was confirmed by western blot and immunofluorescence using the individual samples, which were consistent with our proteomic data. Additionally, an increased rate of the spontaneous acrosome reaction rate was found in the R-ICSI pregnancy group.

Conclusions: The sealtered sperm proteins and the increased spontaneous acrosome reaction rate might account for this unexplained male infertility in the R-ICSI pregnancy patients. The present proteomic results will throw light on the better understanding of the unexplained infertile mechanisms underlying these normozoospermic man who achieved R-ICSI pregnancy after IVF failure.
\end{abstract}

Keywords: Male unexplained infertility, Sperm proteins, iTRAQ, IVF pregnancy, R-ICSI pregnancy

\footnotetext{
*Correspondence: 18615023865@163.com; Ifjyt@126.com

${ }^{\dagger}$ Xin Liu, Gensheng Liu, Juan Liu, and Peng Zhu contributed equally to

this work

${ }^{1}$ Central Laboratory, The Affiliated Yantai Yuhuangding Hospital

of Qingdao University, Yantai 264000, Shandong, People's Republic

of China

${ }^{4}$ Reproductive Center, Beijing BaoDao Obstetrics and Gynecology

Hospital, Beijing 100000, People's Republic of China

Full list of author information is available at the end of the article
}

(c) The Author(s) 2018. This article is distributed under the terms of the Creative Commons Attribution 4.0 International License (http://creativecommons.org/licenses/by/4.0/), which permits unrestricted use, distribution, and reproduction in any medium, provided you give appropriate credit to the original author(s) and the source, provide a link to the Creative Commons license, and indicate if changes were made. The Creative Commons Public Domain Dedication waiver (http://creativecommons.org/ publicdomain/zero/1.0/) applies to the data made available in this article, unless otherwise stated. 


\section{Background}

Approximate $15 \%$ of couples are suffered from infertility, and male factors contribute to $50 \%$ of infertile cases. Male infertility is usually characterized with abnormal sperm motility, concentration, and morphology, which is respectively defined as asthenozoospermia, oligozoospermia, and teratozoospermia. Although the etiology of male infertility is still unclear, ICSI is an effective treatment for man with severe infertility. Additionally, IVF is also used for men with normal semen parameters. In the conventional IVF, a few normozoospermic men experience unexplained fertilization failures with a significant decrease of sperm binding to the zona pellucid $[1,2]$. Considering the normal maturity, number, and quality of retrieved oocytes, male factors might be the major infertile causes, which has been called 'hidden' male factor infertility [3].

A few clinical outcomes indicate the conventional assessment of semen quality cannot satisfy the requirements for the diagnosis of sperm dysfunction $[4,5]$. In order to disclose the unexplained male infertility, proteomic technology has been performed to define the potential sperm proteins in male reproduction. In 2004, two-dimensional electrophoresis has been used to explore the altered sperm protein from a patient experienced failed fertilization during IVF treatment. Compared with the fertile donors, two up-regulated sperm proteins are identified as prolactin-inducible protein and outer dense fiber protein 2 [6]. Anotherproteomic analysis of spermatozoa from patients with no sperm bound to the zona pellucida has been carried out by two-dimensional fluorescence difference gel electrophoresis. Seventeen differentially expressed sperm proteins are identified by mass spectrometry, which might provide the potential biomarkers for the diagnosis and prognosis of the IVF-failed infertility [7]. LC-MS method has also been applied to reveal the differential sperm proteins in the IVF-failure group versus the fertile group. A list of sperm proteins may provide the new understanding of the mechanisms of the IVF failed patients [8].

The present study aimed to deeply reveal sperm protein defects associated with failed fertilization at conventional IVF. The normozoospermic man who achieved fertilization by the IVF or R-ICSI treatment were included, and all the subsequent pregnancies of their female partners were confirmed by an ultrasound detection of fetal heartbeat 6 weeks after embryo. We performed the quantitative proteomic analysis using iTRAQ labeling and LC-MALDI mass spectrometry to disclose differential sperm proteins between the IVF pregnancy group and the R-ICSI pregnancy group. All findings will provide the potential biomarkers for the diagnosis of male infertility and facilitate the better understanding of male reproductive processes.

\section{Methods}

Patients enrollment and sperm sample preparation

According to World Health Organization (WHO) guidelines (WHO Laboratory Manual for the Examination and Processing of Human Semen, 5th) [9], the semen samples included in this study were from 20 normozoospermic men with IVF pregnancy and 20 normozoospermic men with R-ICSI pregnancy. Their female partners met the following criteria: aged no more than 38 years, normal body mass index (BMI) $\left(18.5 \leq \mathrm{BMI} \leq 23.9 \mathrm{~kg} / \mathrm{m}^{2}\right)$ [10-12], without chromosomal abnormalities, endocrine disease, endometriosis or polycystic ovary syndrome (PCOS). Ovulation stimulation were with the long protocol in their first fresh egg retrieval cycle. After IVF or R-ICSI treatment, an ultrasound detection of fetal heartbeat 6 weeks after embryo was used to confirm their pregnancy. After $30 \mathrm{~min}$ of liquefaction, the semen samples were centrifuged at $300 \times g$ for $20 \mathrm{~min}$ to separate spermatozoa from seminal plasma in a $45-90 \%$ discontinuous SpermGrad (Vitrolife, Göteborg, Sweden) gradient. After washing twice in phosphate-buffered saline, the pellet was collected by $450 \times g$ centrifugation for $10 \mathrm{~min}$. The proteins were solubilized in $8 \mathrm{M}$ urea and protease Inhibitor Cocktail (Roche Diagnostics, Oslo, Norway). After centrifugation $\left(20,000 \times g, 30 \mathrm{~min}, 4{ }^{\circ} \mathrm{C}\right)[13,14]$, the protein supernatant was collected and its concentration was determined by the Bradford method [15]. This work has been approved by the Ethics Committee of Beijing BaoDao Obstetrics and Gynecology Hospital, and written informed consents were obtained from all participants.

\section{Spontaneous acrosome reaction measurement}

According to the previous method, spontaneous acrosome reaction was assessedby using FITC-PSA (SigmaAldrich, Steinheim, Germany). Briefly, the above resultant sperm pellet was placed on gelatin-coated slides, and fixed with methanol for $10 \mathrm{~min}$. After incubation with FITC-PSA at room temperature in the dark for $30 \mathrm{~min}$, propidium iodide counterstaining visualized the sperm nuclei. More than 200 cells on each section were examined to calculate the percentage of acrosomereacted sperm under a confocal laser scanning microscope (LSM-510 META; Carl Zeiss, Jena, Germany) [16-18].

\section{ITRAQ analysis of sperm samples}

Equal amounts of sperm proteins from 20 normozoospermic men of IVF pregnancy group, and 20 normozoospermic men of R-ICSI pregnancy group were pooled, and the resultant samples from the IVF or R-ICSI 
pregnancy group were respectively separated into 2 tubes (100 $\mu$ g each) with the $\mathrm{pH}$ adjusted to 8.5. Furtherly, the samples were treated with $20 \mathrm{mM}$ dithiothreitol (DTT) at $56{ }^{\circ} \mathrm{C}$ for $60 \mathrm{~min}$, and $50 \mathrm{mM}$ iodoacetamide (IAA) in the dark for $30 \mathrm{~min}$. After the samples were digested by trypsin (sequencing grade, Promega, France), the tryptic peptides were labelled by iTRAQ reagents, and then dried by a SPD2010 SpeedVac concentrator system (Thermo, North Carolina, USA) [19].

\section{1st pH 10.0 RPLC separation}

The first dimension RP separation was carried out with a Durashell RP column $(5 \mu \mathrm{m}, 150 \AA, 250 \mathrm{~mm} \times 4.6 \mathrm{~mm}$ i.d., Agela, Tianjin, China) on the PF-2D HPLC System (BeckmanCoulter, Fullerton, California, USA). A gradient elution was achieved by mobile phase A ( $2 \%$ acetonitrile, adjusted $\mathrm{pH}$ to 10.0 using $\left.\mathrm{NH}_{3} \cdot \mathrm{H}_{2} 0\right)$ and $\mathrm{B}(98 \%$ acetonitrile, adjusted $\mathrm{pH}$ to 10.0 using $\mathrm{NH}_{3} \cdot \mathrm{H}_{2} \mathrm{O}$ ) at an eluent flow rate of $0.8 \mathrm{ml} / \mathrm{min}$ [20]. Twenty fractions of above labelled peptides were collected and dried by a SPD2010 SpeedVac concentrator system (Thermo, North Carolina, USA).

\section{2nd pH 3.0 RPLC coupled with MALDI Mass Spectrometry}

Briefly, a linear gradient formed by buffer A ( $2 \%$ acetonitrile, $0.1 \%$ formic acid) and buffer B (98\% acetonitrile, $0.1 \%$ formic acid) was used to separate the above fractions at a flow rate of $0.5 \mu \mathrm{L} / \mathrm{min}$. Then the eluted peptides were mixed with matrix solution $(5 \mathrm{mg} / \mathrm{mL}$ in $70 \%$ acetonitrile, $0.1 \%$ trifluoroacetic acid) pushed by additional syringe pump, and spotted on the MALDI plate (AB SCIEX, Massachusetts, USA) using the Tempo ${ }^{\text {TM }}$ LC-MALDI Spotting System (AB SCIEX, California, USA). The 616 spots on each plate were analyzed by a 5800 MALDI-TOF/TOF mass spectrometry (AB SCIEX, Massachusetts, USA). The top 40 ions of the full-scan MS ( $\mathrm{m} / \mathrm{z}$ range from 800 to 4000 ) were chosen for further tandem MS/MS sequencing [19].

\section{Identification and quantification of sperm proteins}

The identification of sperm protein was done with the ProteinPilot $^{\mathrm{TM}}$ software (version 4.0.1; AB SCIEX). A reviewed UniProtKB/Swiss-Prot database (2017_12 released human database, 20,243 entries) was used to search the tandem MS/MS spectrum, and a decoy database (programmed in the ProteinPilot ${ }^{\mathrm{TM}}$ software) was applied to control the data quality $($ FDR $<0.01)$. The searching parameters were iTRAQ 4plex mode, trypsin enzyme, carbamidomethyl cysteine, maximum allowed missed cleavages 1 , and biological modifications programmed in the algorithm. Protein abundances were average ratios of all quantified peptides calculated by the areas of the monoisotopic peaks. The average ratio of four pairs $(116: 114,117: 114,116: 115$, and 117:115) in two repeat experiments was used to determine the statistical alteration of sperm proteins with a confidence interval of 95\% ( $P$ value $<0.05)[19,21]$.

\section{Bioinformatic analysis}

Altered expressed sperm proteins were characterized by the online PANTHER (Protein Analysis THrough Evolutionary Relationships) (released 13.1, 2018-02-03) (http:// pantherdb.org/) [22], DAVID (The Database for Annotation, Visualization and Integrated Discovery; released 6.8, 2016-10) (https://david.ncifcrf.gov/) [23], and the literatures from Pubmed (https://www.ncbi.nlm.nih. gov/pubmed). Each protein was classified into only one category. The protein-protein interaction network was established by the STRING (search tool for recurring instances of neighbouring genes; released 10.5, 2017-0514) (http://string-db.org/) [24].

\section{Western blot analysis}

Fifty $\mu$ g sperm proteins were separated by the SDS-PAGE gel, and then transferred to the nitrocellulose membrane. After blocking with the skimmed milk, and incubating with the primary antibody (Anti-ZPBP1, ab97691, Abcam, Cambridge, USA; Anti-ACRBP, ab64809, Abcam, Cambridge, USA) and HRP-conjugated secondary antibody (ZDR-5306, Zhong-Shan Biotechnology, Beijing, China), the immune-reactive proteins on membrane were visualized by chemiluminescence reagents (Pierce, Rockford, IL, USA). The bands were scanned with a Z320 scanner (Founder, Beijing, China), and analyzed using the ImageJ software (http://imagej.nih.gov/ij/).

\section{Immunofluorescence quantification of spermatozoa protein expression}

Briefly, the above sperm pellet was placed on gelatincoated slides, fixed with methanol, and blocked with BSA. After incubating with the primary antibody (AntiZPBP1, ab97691, Abcam, Cambridge, USA) and the FITC-labelled secondary antibody (ZF-0311, Zhongshan golden bridge biotechnology, Beijing, China), all sections were mounted with glycerol and determined with a LSM-510 META confocal laser scanning microscope (Carl Zeiss, Jena, Germany). The intensity and percent of spermatozoa with positive Immunofluorescence staining were assessed according to the previous method [25].

\section{Statistical analysis}

The data of two group means in this study were calculated using the unpaired $t$ test with $P<0.05$ of statistical significance. All analysis was performed with software of 
Statistical Package for the Social Sciences (SPSS v. 18.0, Chicago, IL, USA).

\section{Results}

Semen parameters of normozoospermic men

In accordance with World Health Organization (WHO) guidelines, all the men recruited in this study were normozoospermic. They had normal BMI, semen volume, total sperm count, progressive motility, sperm concentration, sperm morphology. Their partners were also characterized with normal BMI, FSH, LH, E2, number of retrieved oocytes, and without PCOS, endometriosis, endocrine disease or chromosomal abnormalities. The statistical analysis showed no differences of these reproductive parameters between the IVF pregnancy group and the R-ICSI pregnancy group (Table 1). Considering the subsequently successful clinical pregnancy and the phenotype with a dramatical decrease of sperm binding to the zona pellucida, male factor was the cause of failed fertilization at IVF, instead of their female partners (Fig. 1).

\section{Spontaneous acrosome reaction}

FITC-PSA was applied to determine the difference of the spontaneous acrosome reactions between the IVF pregnancy group and R-ICSI pregnancy group. In comparison with sperm from the normozoospermic men of IVF pregnancy group, the percentage of acrosome-reacted sperm showed a non-significant increase in normozoospermic men of R-ICSI group ( $P=0.0756)$ (Fig. 2$)$.
Comparative analysis of normozoospermic sperm proteins between the IVF pregnancy group and the R-ICSI pregnancy group

Figure 3 indicates the flowchart of iTRAQ-based proteomic analysis of normozoospermic sperm from IVF and R-ICSI pregnancy group. Briefly, the sperm proteins were digested by trypsin, labeled by iTRAQ reagents, separated by two-dimension RPLC, spotted on the MALDI plate, and sequenced by mass spectrometry. The ProteinPilot $^{\mathrm{TM}}$ software was applied to analyze the spectraby searching the reviewed Swiss-Prot human database (20,316 sequences, 2018_02 released). A total of 450 sperm proteins were identified and quantified with a high confidence (FDR < 0.01) (Additional file 1: Supplementary Table 1). Compared with the IVF pregnancy group, 36 sperm proteins were up-regulated $\left(\right.$ ratio $_{\mathrm{R}-\mathrm{ICSI} / \mathrm{IVF}}>1.274$, $P<0.05)$ (Table 2), and 20 sperm proteins were downregulated $\left(\right.$ ratio $_{\mathrm{R}-\mathrm{ICSI} / \mathrm{IVF}}<0.762, P<0.05$ ) (Table 3 ) in the R-ICSI pregnancy group. Figure 4 shows the representative MS/MS spectrum of iTRAQ-labelled peptide from zona pellucida-binding protein 1 noted with most b-ions and y-ions.

\section{GO classification of altered sperm proteins associated} with R-ICSI pregnancy after conventional failed IVF Based on PANTHER, DAVID and the PubMed literature, the differentially expressed sperm proteins were classified into GO categories (Fig. 5). As for higher abundant sperm proteins in the R-ICSI pregnancy group, protein binding (36\%) and nucleic acid binding (22\%) were the main molecular functions; nucleus (20\%) and membrane

Table 1 Semen analysis and IVF cycle data (Mean \pm SEM)

\begin{tabular}{|c|c|c|c|}
\hline Characteristics & $\begin{array}{l}\text { Normozoospermic men with IVF } \\
\text { pregnancy }^{\mathrm{a}}\end{array}$ & $\begin{array}{l}\text { Normozoospermic men with R-ICSI } \\
\text { pregnancy }^{\mathrm{a}}\end{array}$ & $P$ value \\
\hline \multicolumn{4}{|l|}{ Semen } \\
\hline Age & $32.1 \pm 0.2$ & $31.6 \pm 0.2$ & 0.125 \\
\hline BMI $\left(\mathrm{kg} / \mathrm{m}^{2}\right)$ & $22.6 \pm 0.1$ & $22.3 \pm 0.1$ & 0.161 \\
\hline Volume $(\mathrm{mL})$ & $2.9 \pm 0.1$ & $3.0 \pm 0.1$ & 0.410 \\
\hline Total sperm count (10\%/ejaculate) & $179.0 \pm 3.2$ & $187.4 \pm 4.1$ & 0.114 \\
\hline Progressive motility (\%) & $37.1 \pm 0.3$ & $37.7 \pm 0.3$ & 0.139 \\
\hline Sperm morphology (normal%) & $7.1 \pm 0.1$ & $7.2 \pm 0.2$ & 0.555 \\
\hline \multicolumn{4}{|l|}{ IVF data } \\
\hline Age & $31.7 \pm 0.2$ & $31.6 \pm 0.2$ & 0.563 \\
\hline BMI $\left(\mathrm{kg} / \mathrm{m}^{2}\right)$ & $21.9 \pm 0.2$ & $21.8 \pm 0.2$ & 0.757 \\
\hline Basal FSH (U/L) & $6.3 \pm 0.1$ & $6.4 \pm 0.1$ & 0.179 \\
\hline Basal LH (U/L) & $3.5 \pm 0.1$ & $3.3 \pm 0.1$ & 0.111 \\
\hline Basal E2 (ng/L) & $32.4 \pm 0.5$ & $32.8 \pm 0.4$ & 0.487 \\
\hline No. of retrieved oocytes & $13.2 \pm 0.3$ & $13.5 \pm 0.3$ & 0.501 \\
\hline No. of 2 pro-nuclear zygotes & $9.2 \pm 0.2$ & $9.3 \pm 0.2$ & 0.658 \\
\hline
\end{tabular}

a An ultrasound detection of fetal heartbeat 6 weeks after embryo 


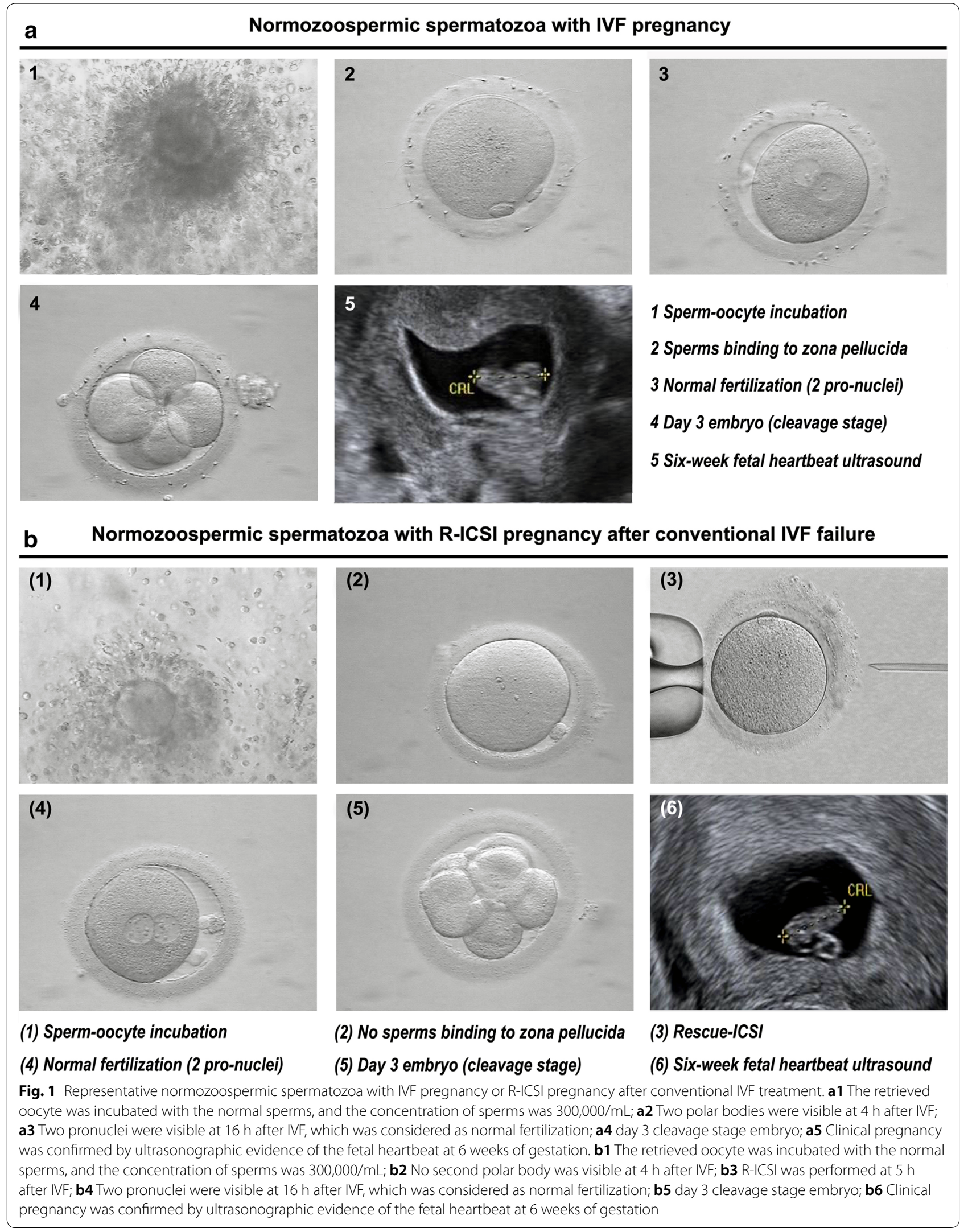



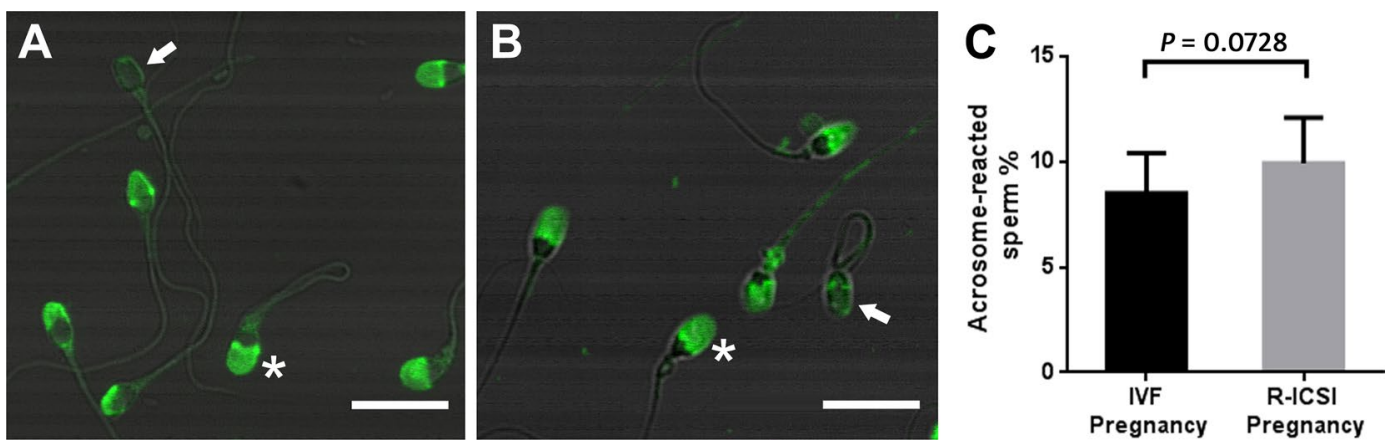

Fig. 2 Representative fluorescence images and quantification of FITC-PSA staining. A The detection of spontaneous acrosome reaction in the IVF pregnancy group; $\mathbf{B}$ The detection of spontaneous acrosome reaction in the R-ICSI pregnancy group; C The quantification of spontaneous acrosome reaction between the IVF-pregnancy group $(n=20)$ and the R-ICSI pregnancy group $(n=20)$. Acrosome-intact sperm is marked by asterisk, and acrosome-reacted sperm is marked by arrow. Each bar indicates $10 \mu \mathrm{m}$

(20\%) were the majority of subcellular localizations; reproduction (20\%), developmental process (19\%), chromosome organization (14\%) were the important biological processed. In the lower abundant sperm proteins in the R-ICSI pregnancy group, sperm-oocyte interaction (20\%) was the main molecular function, membrane (30\%) accounted for a large portion of subcellular localization, and reproduction (25\%) and development process (15\%) were the main biological processed.

\section{Interaction Networks of sperm proteins involved in the R-ICSI pregnancy after conventional IVF failure}

The online STRING software was applied to construct the protein-protein interaction network of the altered sperm proteins related to the R-ICSI pregnancy after failed IVF treatment (Fig. 6). Forty differentially expressed proteins were classified into three interaction groups. First group included 3 sperm proteins (ADP-ribosylation factor 5, dynein light chain 1, and dynein light chain 2), second group consisted of 6 sperm proteins (long chain fatty acid CoA ligase 6, hemoglobin subunit alpha, retinal dehydrogenase 1 , very long chain specific acyl CoA dehydrogenase, electron transfer flavoprotein subunit beta, and 3-hydroxyisobutyrate dehydrogenase), and third group covered the left 31 proteins (zona pellucida-binding protein 1, acrosin-binding protein, spermatid maturation protein 1, izumo sperm-egg fusion protein 4, tubulin alpha-3C/D chain, peroxiredoxin-1, peptidyl-prolyl cis-trans isomerase $\mathrm{A}$, protein disulfide-isomerase A3, serum amyloid P-component, actin-related protein $\mathrm{T} 3$, histone H2A type 1-A, zinc finger RNA-binding protein, histone H3.2, izumo sperm-egg fusion protein 3, $26 \mathrm{~S}$ proteasome non-ATPase regulatory subunit 2, histone $\mathrm{H} 4$, proteasome subunit beta type-2, afamin, hyaluronidase $\mathrm{PH}-20$, proteasome subunit alpha type-1, zinc-alpha2 -glycoprotein, alpha-1-acid glycoprotein 2 , protamine-2,
alpha-1B-glycoprotein, hemopexin, transthyretin, alpha2-HS-glycoprotein, alpha-1-acid glycoprotein 1, protein AMBP, beta-2-glycoprotein 1, glyceraldehyde-3-phosphate dehydrogenase).

\section{Comparison of present sperm proteome and the previous reported IVF-related sperm proteomes}

To indicate the overlap of the IVF-related sperm proteomes between different labs and to explore the new findings, the previous published sperm profiles associated with the different outcome under the IVF treatment were selected $(7,8)$, and Fig. 7 shows the overlap between them. Compared with the present work, approximate 90\% IVF-related sperm proteins were not identified in the previous researches.

\section{Western blot analysis}

To validate theconfidence of iTRAQ technology for protein quantification, western blot was used to analyze the expression patterns of zona pellucida-binding protein 1 (ZPBP1) and acrosin-binding protein (ACRBP) by the individual samples (Fig. 8). The outcome indicated the levels of ZPBP1 and ACRBP were significantly lower in the R-ICSI pregnancy group than in the IVF pregnancy group $(P$ value $<0.01)$, which were consistent with the proteomic results.

\section{Immunofluorescence quantification}

Further immunofluorescence analysis demonstrated that zona pellucida-binding protein 1 was located on the acrosome region of spermatozoa from IVF pregnancy group and R-ICSI pregnancy group. But the percent of spermatozoa with positive staining and fluorescence intensity were statistically decreased in normozoospermic men with R-ICSI pregnancy compared to normozoospermic men with IVF pregnancy $(P<0.05$, Fig. 9). 


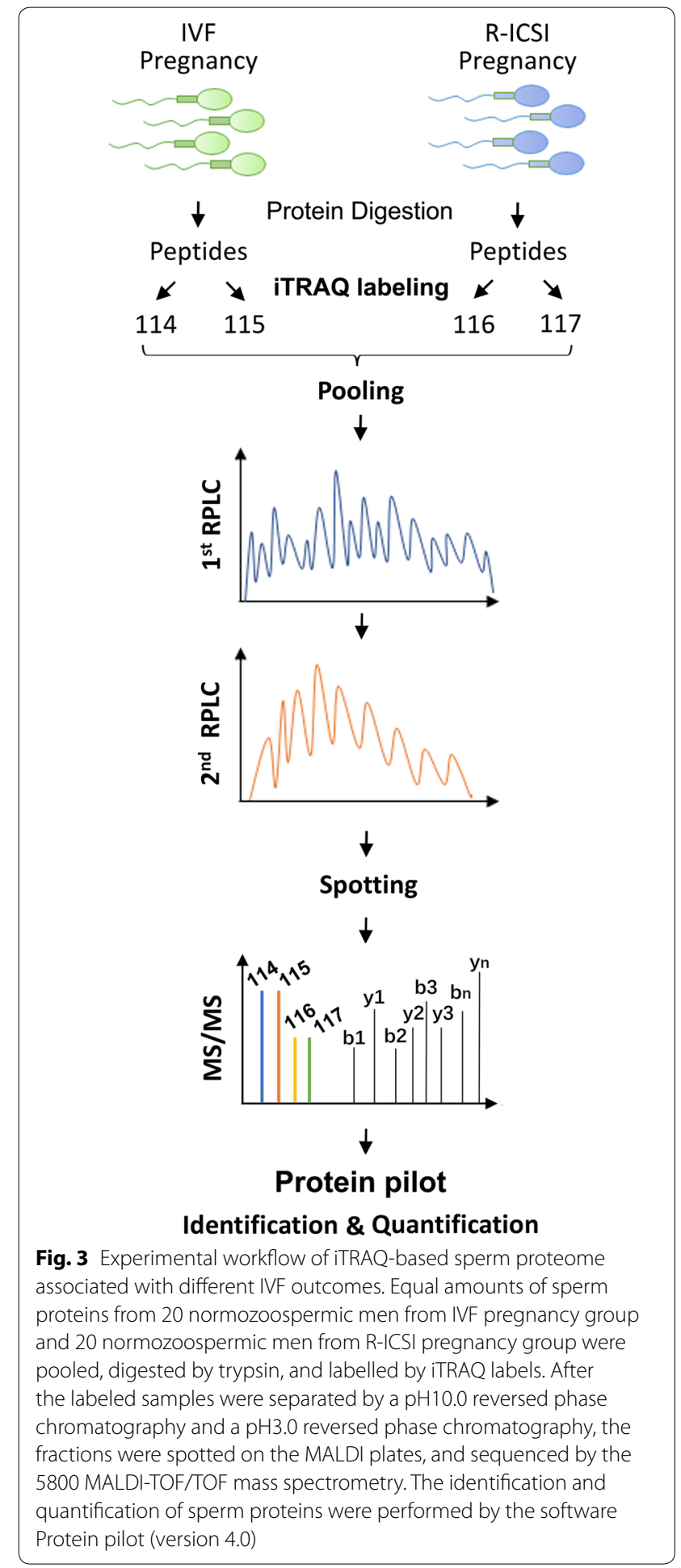

\section{Table 2 Higher abundant sperm proteins in normozoospermic samples that achieved pregnancy undergoing R-ICSI after IVF failure $(n=36)$}

Swiss-Prot Protein name Ratio (Mean \pm SD)

accession

number IVF pregnancy

\begin{tabular}{lll}
\hline P62805 Histone H4 2.62 & &
\end{tabular}

095996 Adenomatous polyposis coli protein $2 \quad 2.29 \pm 0.12$

Q8NCQ7 Protein PROCA1 $2.13 \pm 0.12$

$\begin{array}{ll}\text { P04554 Protamine-2 } & 1.97 \pm 0.12\end{array}$

$\begin{array}{lll}\text { P19652 Alpha-1-acid glycoprotein } 2 & 1.93 \pm 0.38\end{array}$

$\begin{array}{ll}\text { P02766 Transthyretin } & 1.77 \pm 0.10\end{array}$

P63167 Dynein light chain $1 \quad 1.72 \pm 0.09$

Q13200 26S proteasome non-ATPase regula- $\quad 1.66 \pm 0.23$ tory subunit 2

P02749 Beta-2-glycoprotein $1 \quad 1.57 \pm 0.11$

P31937 3-hydroxyisobutyrate dehydrogenase $\quad 1.56 \pm 0.01$

014556 Glyceraldehyde-3-phosphate dehydro- $1.55 \pm 0.02$ genase, testis-specific

$\begin{array}{ll}\text { P02763 Alpha-1-acid glycoprotein } 1 & 1.54 \pm 0.21\end{array}$

$\begin{array}{ll}\text { P02760 Protein AMBP } & 1.53 \pm 0.04\end{array}$

$\begin{array}{lll}\text { P55064 Aquaporin-5 } & 1.53 \pm 0.11\end{array}$

$\begin{array}{lll}\text { Q9Y4G6 Talin-2 } & 1.52 \pm 0.20\end{array}$

$\begin{array}{lll}\text { P38567 Hyaluronidase PH-20 } & 1.51 \pm 0.07\end{array}$

Q6UWM5 GLIPR1-like protein 1 $\quad 1.49 \pm 0.13$

P25786 Proteasome subunit alpha type-1 $\quad 1.48 \pm 0.11$

$\begin{array}{lll}\text { Q9BYD9 Actin-related protein T3 } & 1.48 \pm 0.16\end{array}$

$\begin{array}{ll}\text { P02765 Alpha-2-HS-glycoprotein } & 1.47 \pm 0.07\end{array}$

$\begin{array}{ll}\text { P04217 Alpha-1B-glycoprotein } & 1.45 \pm 0.10\end{array}$

P02790 Hemopexin $1.44 \pm 0.08$

$\begin{array}{lll}\text { P25311 Zinc-alpha-2-glycoprotein } & 1.44 \pm 0.08\end{array}$

P49748 Very long-chain specific acyl-CoA $\quad 1.43 \pm 0.08$

Q68DN1 Uncharacterized protein C2orf16 $\quad 1.42 \pm 0.13$

Q9BZW7 Testis-specific gene 10 protein $\quad 1.40 \pm 0.03$

Q96FJ2 Dynein light chain 2 $\quad 1.39 \pm 0.03$

Q71DI3 Histone H3.2 1.39 \pm 0.10

A6NMS7 Leucine-rich repeat-containing protein $1.39 \pm 0.07$

$\begin{array}{lll}\text { P43652 Afamin } & 1.32 \pm 0.03\end{array}$

Q8N0W7 Fragile X mental retardation 1 neigh- $\quad 1.32 \pm 0.14$ bor protein

$\begin{array}{lll}\text { P49721 Proteasome subunit beta type-2 } & 1.31 \pm 0.07\end{array}$

Q5VZ72 Izumo sperm-egg fusion protein $3 \quad 1.30 \pm 0.13$

Q96QV6 Histone H2A type 1-A $1.29 \pm 0.22$

Q96KR1 Zinc finger RNA-binding protein $\quad 1.29 \pm 0.10$

P38117 Electron transfer flavoprotein subunit $1.28 \pm 0.12$ beta

Annotations of altered proteins identified in sperm samples from the R-ICSI pregnancy group compared with the IVF pregnancy group. Values $>1.274$ $(P<0.05)$ correspond to higher abundance in the R-ICSI pregnancy group. Standard deviation (SD) 
Table 3 Lower abundant sperm proteins in normozoospermic samples that achieved pregnancy undergoing R-ICSI after IVF failure $(\mathbf{n}=\mathbf{2 0})$

\begin{tabular}{lll}
\hline Swiss-Prot accession number & Protein name & $\begin{array}{l}\text { Ratio (Mean } \pm \text { SD) R-ICSI pregnancy/ } \\
\text { IVF pregnancy }\end{array}$ \\
\hline P62937 & Peptidyl-prolyl cis-trans isomerase A & $0.76 \pm 0.01$ \\
Q5VTE0 & Putative elongation factor 1-alpha-like 3 & $0.76 \pm 0.07$ \\
Q9BXU7 & Ubiquitin carboxyl-terminal hydrolase 26 & $0.76 \pm 0.08$ \\
Q9BS86 & Zona pellucida-binding protein 1 & $0.75 \pm 0.02$ \\
P02743 & Serum amyloid P-component & $0.75 \pm 0.02$ \\
Q9UKU0 & Long-chain-fatty-acid-CoA ligase 6 & $0.75 \pm 0.16$ \\
Q8N4L4 & Spermatid maturation protein 1 & $0.74 \pm 0.03$ \\
Q8NEB7 & Acrosin-binding protein & $0.74 \pm 0.09$ \\
Q13748 & Tubulin alpha-3C/D chain & $0.74 \pm 0.04$ \\
P30101 & Protein disulfide-isomerase A3 & $0.74 \pm 0.05$ \\
P69905 & Hemoglobin subunit alpha & $0.72 \pm 0.03$ \\
Q1ZYL8 & Izumo sperm-egg fusion protein 4 & $0.71 \pm 0.01$ \\
Q6NXR0 & Interferon-inducible GTPase 5 & $0.70 \pm 0.02$ \\
P07477 & Trypsin-1 & $0.70 \pm 0.10$ \\
P84085 & ADP-ribosylation factor 5 & $0.68 \pm 0.13$ \\
Q13642 & Four and a half LIM domains protein 1 & $0.67 \pm 0.05$ \\
Q499Z3 & Schlafen-like protein 1 & $0.61 \pm 0.06$ \\
P00352 & Retinal dehydrogenase 1 & $0.61 \pm 0.11$ \\
Q06830 & Peroxiredoxin-1 & $0.60 \pm 0.12$ \\
Q96C74 & Ropporin-1-like protein & $0.55 \pm 0.07$
\end{tabular}

Annotations of altered proteins identified in sperm samples from the R-ICSI pregnancy group compared with the IVF pregnancy group. Values $<0.762(P<0.05)$ correspond to lower abundance in the R-ICSI pregnancy group. Standard deviation (SD)

\section{Discussion}

Hitherto, there is still unclear about the underlying mechanisms of the unexplained infertility in the normozoospermic men. In the R-ICSI group, although the parameters of retrieved oocytes were normal, there was still a lack of sperm fixation to the zona pellucida. Meanwhile, to exclude the infertile factors from their female partners as far as possible, the subsequent pregnancies were all confirmed by an ultrasound detection of 6-week fetal heartbeat after fertilization by IVF or R-ICSI treatment in this study. Therefore, we provided a hypothesis of the sperm-oocyte interaction molecule defects underlying this unexplained male factor infertility.

To discover the critical infertile molecules, the proteomic technique including two-dimensional electrophoresis has been widely used to realize these goals [6-8]. Compared with the previous conventional proteomic researches, the powerful iTRAQ based quantitative proteomics was applied to reveal more altered sperm proteins in this work. The overlap of the IVF-related sperm proteomes indicated only 5 of 56 differential proteins have been identified and quantified such as protein disulfide-isomerase A3, histone $\mathrm{H} 3.2$, histone $\mathrm{H} 4$, glyceraldehyde-3-phosphate dehydrogenase, and tubulin alpha-3C/D chain in the overlap. The low common identification of sperm proteins might be mainly caused by different experimental design, patient recruitment, sperm handling, quantitative methods, and data processing. in the future, the collaboration of international laboratories should be established to provide the better understanding on male infertility.

Through the analysis of the online STRING software three protein-protein interaction networks were delineated with 40 altered sperm proteins involved in R-ICSI pregnancy. The biggest interaction network contained 31 nodes including several down-regulated proteins associated with sperm-oocyte interaction, e.g. acrosinbinding protein (ACRBP) and zona pellucida-binding protein 1 (ZPBP1), which displayed the complexity of 


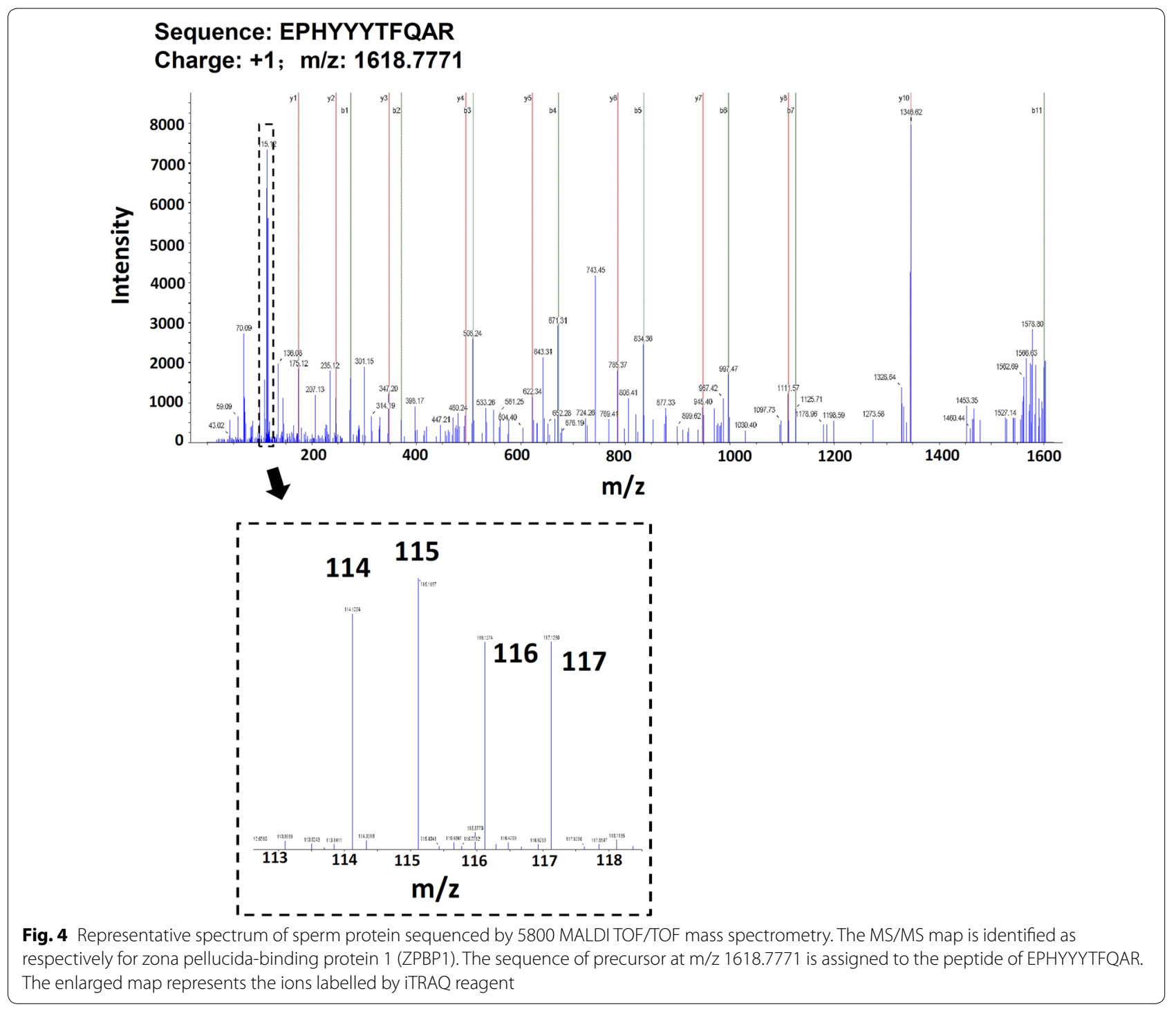

the mechanisms under the male infertility. ACRBP is a proacrosin binding protein and participate in the process of the acrosin zymogen condensation [26], and ZPBP1 also plays an important role in acrosome compaction [27]. ACRBP and ZPBP1 has been identified as high molecular weight complexes from sperm head plasma membrane and defined as the acrosomal proteins. As the associated partner of proacrosin (with zona pellucida affinity), the transportation of ACRBP from acrosome to sperm head surface seems to be involved in the initial binding between sperm head surface and zona pellucida, which subsequently induce the acrosome reaction. 


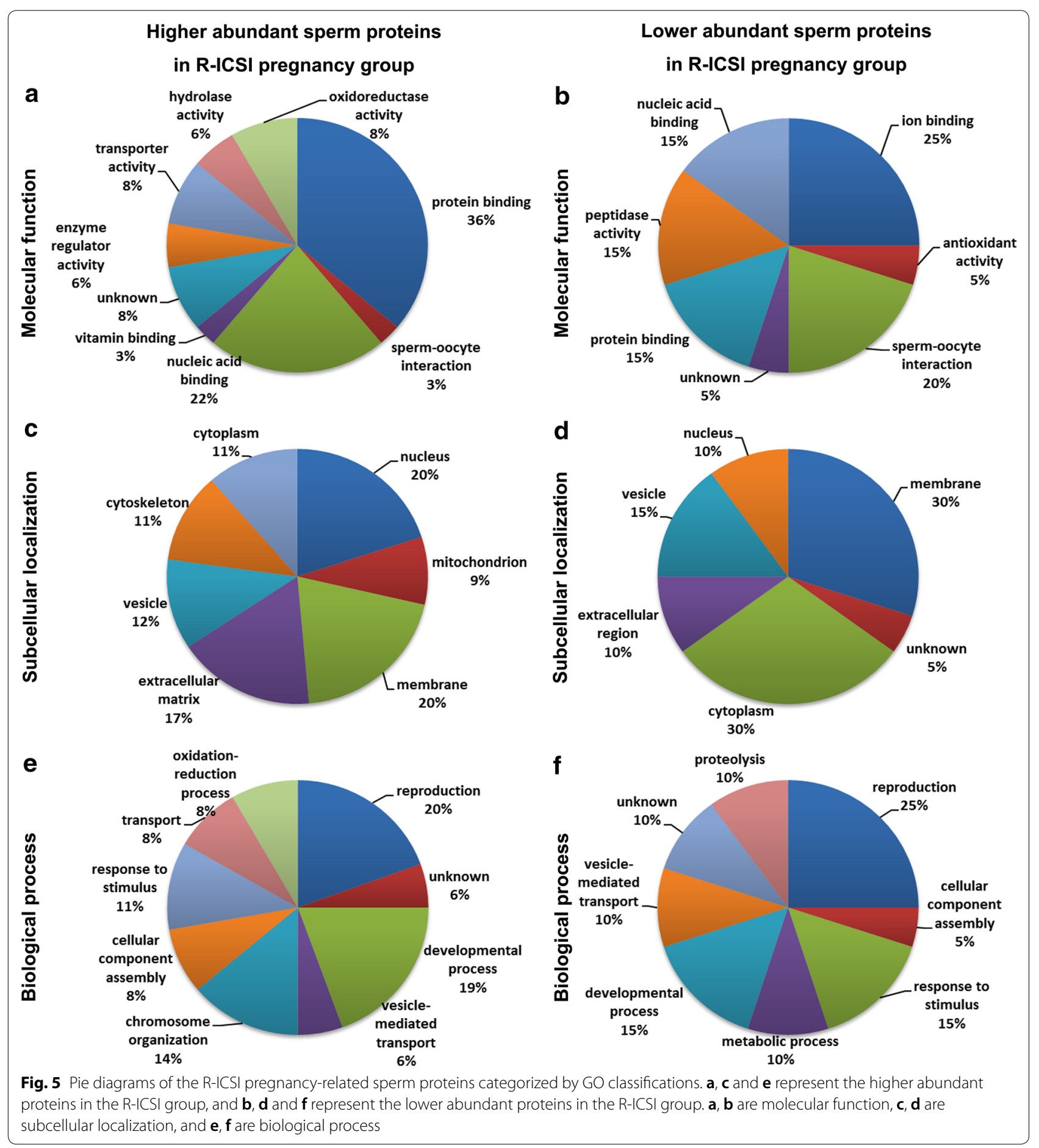




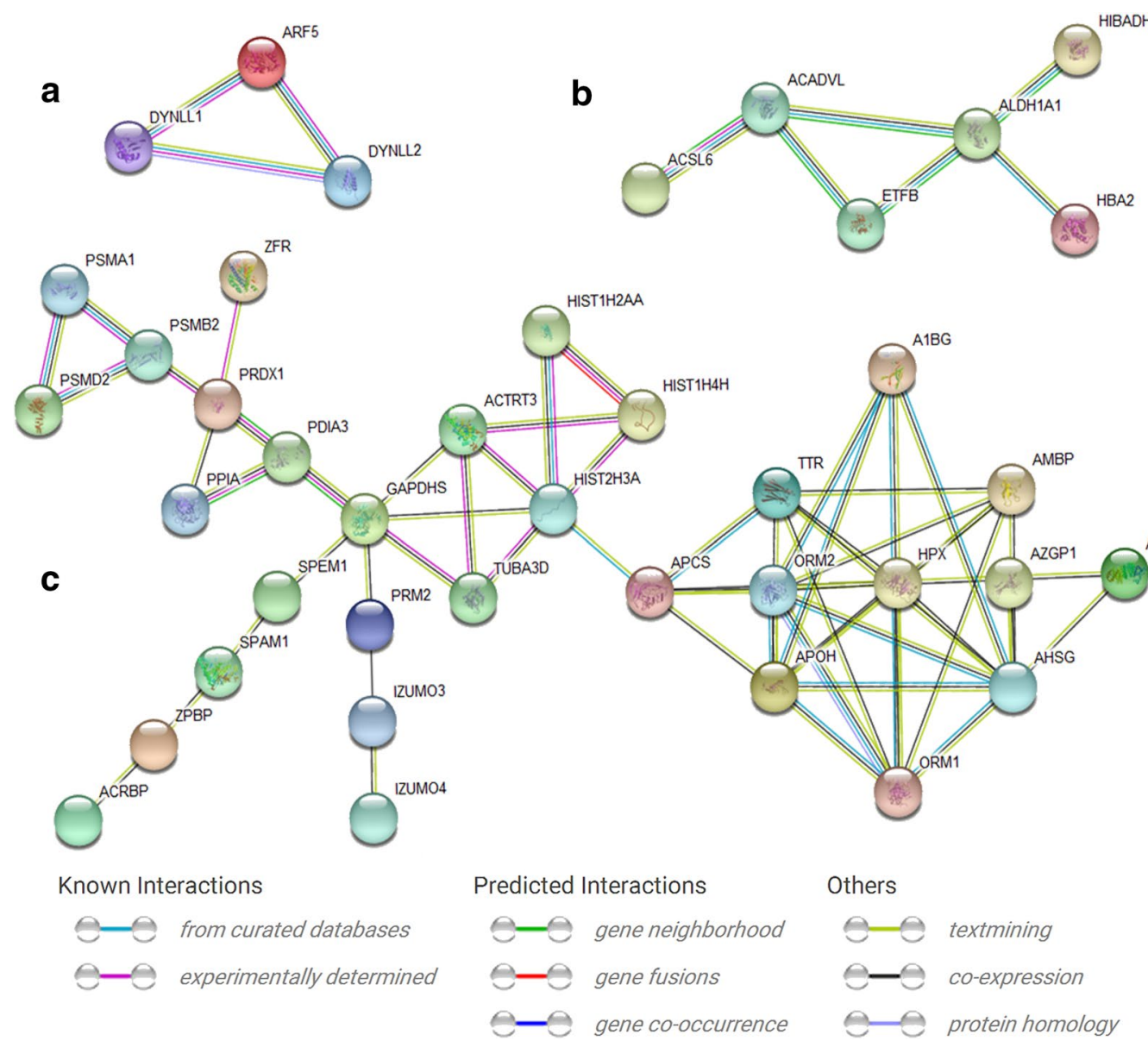

Fig. 6 Representative protein-protein interaction networks of altered R-ICSI pregnancy-related sperm proteins. A total of 40 genes was classified into 3 clusters. a Cluster contained three nodes, $\mathbf{b}$ cluster included six nodes, and $\mathbf{c}$ cluster was consisted of 31 nodes. The relationships were derived from the curated databases, experimentally determined, gene neighborhood, gene fusions, gene co-occurrence, textmining, co-expression, and protein homology

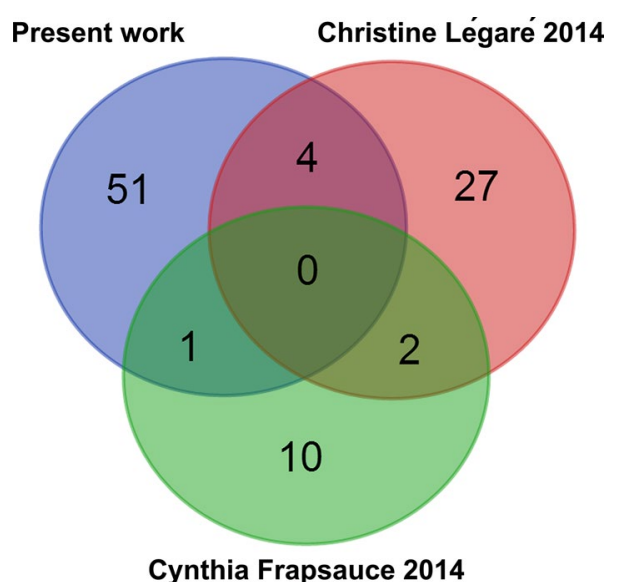

Fig. 7 Venn diagram of the overlaps of the present work and the other two differential IVF-related sperm proteomes (Légaré et al. [8], Frapsauce et al. [7])
During the secondary sperm binding, the exposed inner acrosomal membranes of the acrosome-reacted sperm further fix to and penetrate the zona pellucida [28]. ZPBP1 isa acrosomal protein with the zona pellucida glycoprotein affinity, which locates on the inner acrosomal membrane of the anterior acrosome, and the inner acrosomal membrane and the outer acrosomal membrane of the equatorial segment. Even after the acrosome reaction, ZPBP1 is still located on the inner acrosomal membrane, which facilitates the secondary sperm binding to zona pellucida and the following zona penetration [29, 30]. Another research has reported that ZPBP1 competes with proacrosin (the binding substrate of ACRBP) for fixation to the zona pellucida during the fertilization process [31]. Recent study indicated ZPBP1 gene mutation is also involved in the teratospermia [32]. In the present work, ACRBP and ZPBP1 were both statistically downregulated in the R-ICSI pregnancy group after the failure 


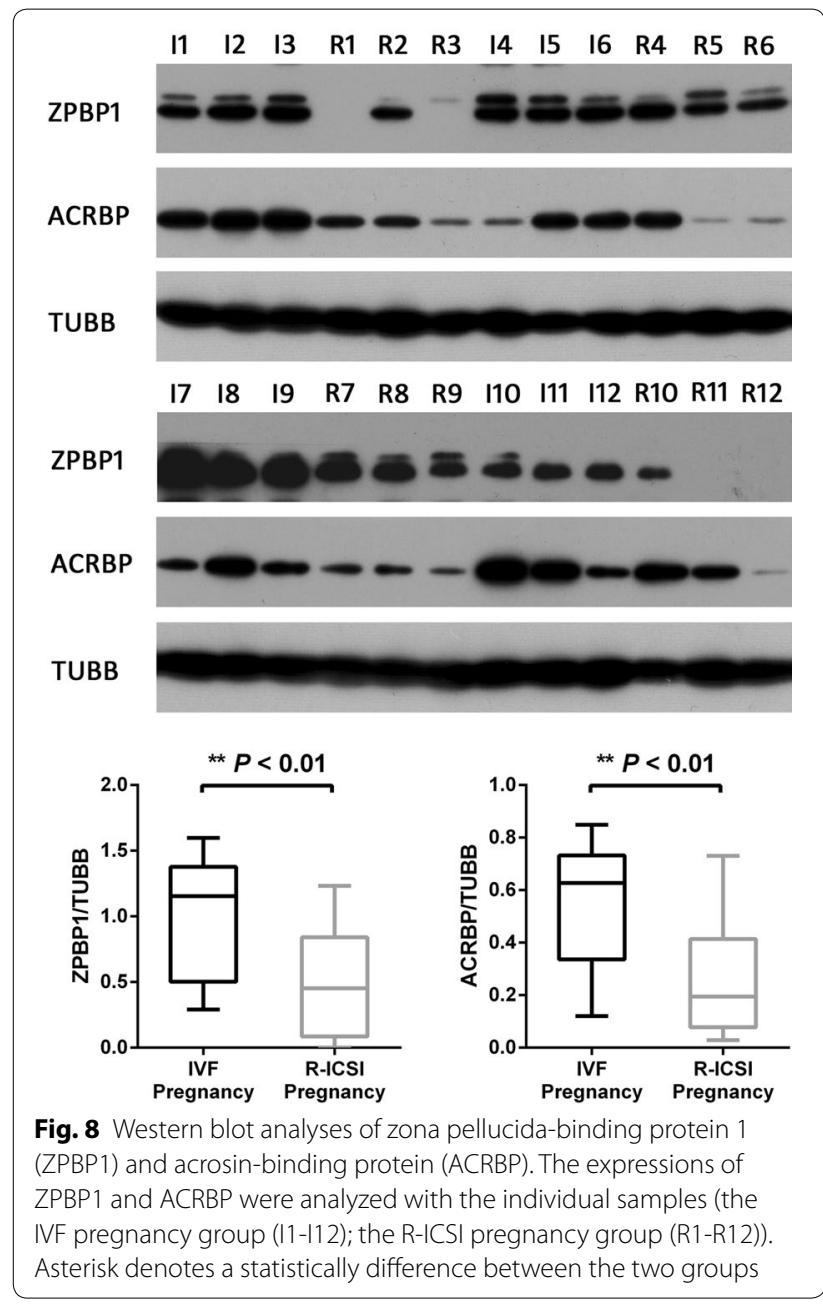

of conventional IVF. Lower ACRBP expression might not only weaken the initial binding to zona pellucida but also increase the level of free proacrosin. The latter would compete with ZPBP1 for binding to zona pellucida. In addition, the decreased ZPBP1 also could attenuate the secondary sperm binding to zona pellucida. As a result, the failed insemination characterized with no binding to the zona pellucida could appear in the previously clinical IVF treatment.

In addition to the above altered sperm proteins, a dramatical decrease of sperm binding to the zona pellucida also indicated that there might be some defects on the acrosome reaction in the R-ICSI pregnancy group. Acrosome is an important organelle for the fertilization. The anterior acrosome was related to sperm-zona pellucida interaction, and the posterior acrosome is associated with sperm-egg fusion [33]. The occurrence of sperm acrosome reaction releases some necessary lysins, which facilitates sperm to penetrate the zona pellucida and fuse with oocyte membrane [34, 35]. That is to say, acrosome reaction should occur after the sperm binding to the zona pellucida. Otherwise, the spontaneous occurrence of acrosome reaction without intact with the zona pellucida will deprive the sperm fertilization potential [36]. But spontaneous acrosome reaction can arise before contact with the zona pellucida, which is associated with the low reproductive potential. It has been reported that the spontaneous acrosome reaction rate is higher in the spermatozoa from obese group than from lean group [37]. Obesity is usually related to a higher risk for male subfertility, which is characterized with the reduced sperm concentration, count and motility [38]. In 1984, Plachot et al. suggested no correlation could be found between the acrosomal reaction rate and the ability to fertilize the oocyte [39]. Recently, spontaneous acrosome reaction has been used to evaluate the semen quality during IVF, which provides a new way to decide whether conventional insemination or ICSI is preferable treatment. As a result, the samples with a high rate of spontaneous acrosome reaction shows a significant low fertilization rate compared with those with a low rate of spontaneous acrosome reaction [40]. In the present work, the BMI index of both IVF-pregnancy group and R-ICSI pregnancy group were normal, and without statistical difference. Therefore, obesity is not cause of the occurrence of spontaneous acrosome reaction. This unexplained higher spontaneous acrosome reaction rate might also be responsible for the failure of conventional insemination.

\section{Conclusions}

In the past decades, IVF has been widely applied to help the couples undergoing the failed natural fertilization. To explore the male infertile molecular causes of the failed IVF (normozoospermic men with a severe decrease of sperm binding to the zona pellucida of the normal oocytes), an iTRAQ proteomic approach was used to reveal the infertility-related differential sperm proteins between the R-ICSI pregnancy group and the conventional IVF pregnancy group men. The new panel of the altered sperm proteins, especially these sperm-oocyte interaction proteins will provide novel clues to understand the mechanisms of male infertility and become the potential biomarkers for the better prediction of IVF outcomes. Further studies are still 


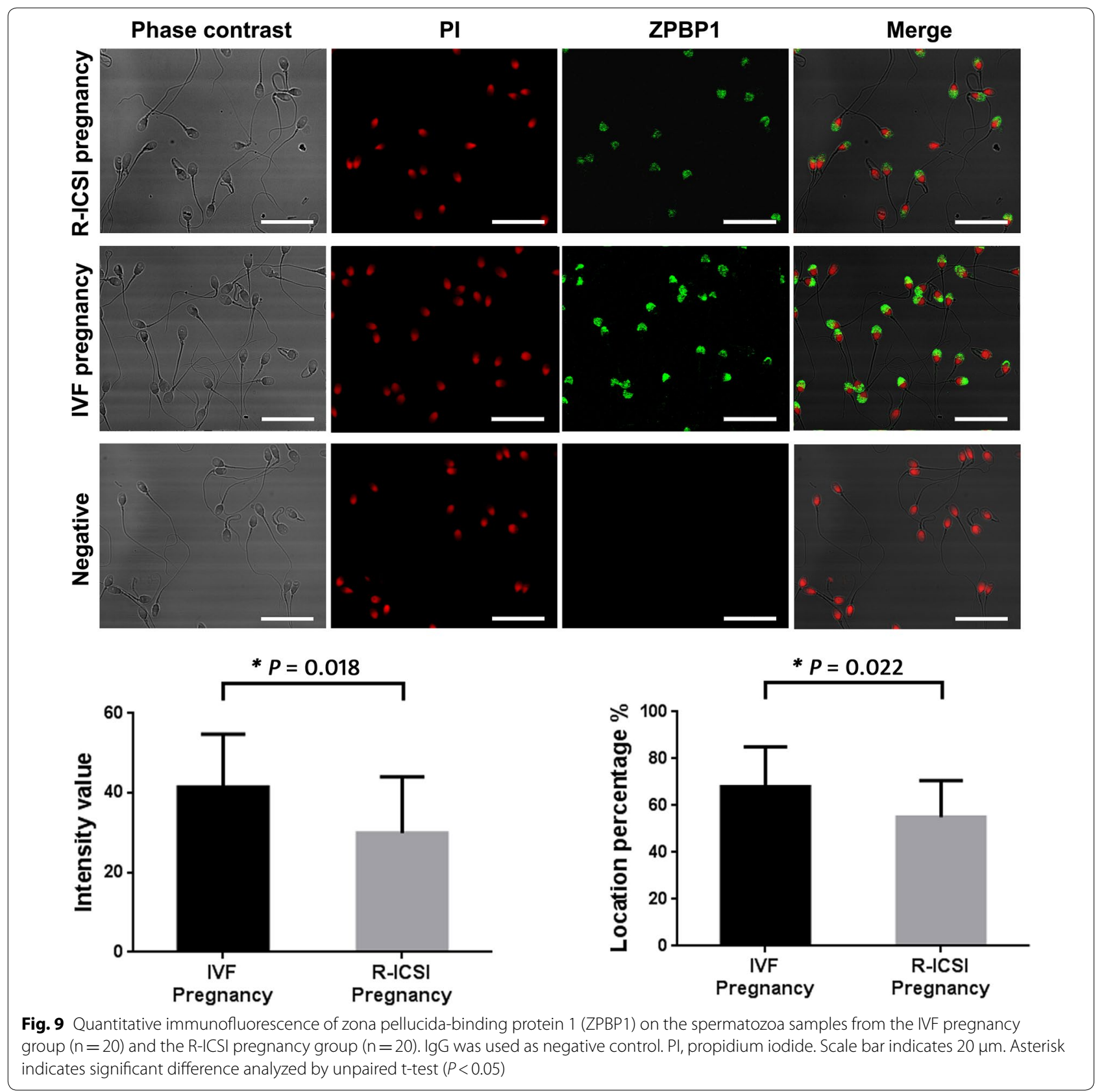

necessary to confirm the feasibility of these potential markers used for the diagnosis and prognosis of male infertility.

\section{Additional file}

Additional file 1: Supplementary Table 1. The quantitative details of sperm proteins from normozoospermic men between the IVF pregnancy group and the R-ICSI pregnancy group.

\section{Abbreviations}

IVF: in vitro fertilization; R-ICSI: rescue intracytoplasmic sperm injection; iTRAQ: isobaric tags for relative and absolute quantification; RPLC: high-performance liquid chromatography; MALDI: matrix-assisted laser desorption/ionization; MS: mass spectrometry; WHO: world health organization; PCOS: polycystic ovary syndrome; FITC: fluorescein isothiocyanate; PSA: pisum sativum agglutinin; PANTHER: protein analysis through evolutionary relationships: DAVID: the database for annotation, visualization and integrated discovery; STRING: search tool for recurring instances of neighbouring genes; BMI: body mass index; FSH: follicle-stimulating hormone; LH: luteinizing hormone; E2: estradiol; FDR: false discovery rate; ACRBP: acrosin-binding protein; ZPBP1: zona pellucida-binding protein 1 . 


\section{Authors' contributions}

$X L, G L, X S$, and FL conceived of the study and participated in its design. $X L, G L$ $X S$, FL, and JW drafted the manuscript. GL and XS performed the sample collection. JL, PZ, YW, WW, NL, XW, and CZcarried out the studies and contributed to the acquisition of data. $\mathrm{XL}$ and FL performed the proteomic analysis. PZ, and CZ carried out the western blot. WW and JL performed immunofluorescence quantification. NL, YW, XW, and FL carried out the bioinformatics analysis. JL, NL carries out the bioinformatics analysis. All authors read and approved the final manuscript.

\section{Author details}

${ }^{1}$ Central Laboratory, The Affiliated Yantai Yuhuangding Hospital of Qingdao University, Yantai 264000, Shandong, People's Republic of China. ${ }^{2}$ Reproductive Center, Tianjin Aiwei Hospital, Tianjin 300011, People's Republic of China. ${ }^{3}$ Department of Clinical Laboratory, The Affiliated Yantai Yuhuangding Hospital of Qingdao University, Yantai 264000, Shandong, People's Republic of China. ${ }^{4}$ Reproductive Center, Beijing BaoDao Obstetrics and Gynecology Hospital, Beijing 100000, People's Republic of China.

\section{Acknowledgements}

We thank Guo Lihai Ph.D. (Shanghai Asia Pacific Application Support Center, Applied Biosystems, China) for the usage training of RPLC-MALDI-TOF/TOF 5800 mass spectrometer (AB SCIEX, USA).

\section{Competing interests}

The authors declare that they have no competing interests.

\section{Availability of data and materials}

The datasets used and/or analyzed during the current study available from the corresponding author on reasonable request.

\section{Consent for publication}

All authors consent to the publication of this manuscript.

\section{Ethics approval and consent to participate}

This work has been approved by the Ethics Committee of Yantai Yuhuangding Hospital, and written informed consents wereobtained from all participants.

\section{Funding}

The current study was supported by the National Natural Science Foundation of China (Grant Nos. 81571490, 81501313, and 81701453), Shandong Provincial Natural Science Foundation, China (Grant No. ZR2014HQ068), the Key Research and Development Program of Shandong Province (Grant No. 2017GSF18163), and Yantai Science and Technology Program (Grant No. 2016WS001).

\section{Publisher's Note}

Springer Nature remains neutral with regard to jurisdictional claims in published maps and institutional affiliations.

Received: 28 May 2018 Accepted: 14 August 2018 Published online: 21 August 2018

\section{References}

1. Neal GM, Aydin A. Failed fertilization: is it predictable? Curr Opin Obstet Gynecol. 2003;15:211-8.

2. Liu DY, Baker HWG. Disordered zona pellucida-induced acrosome reaction and failure of invitro fertilization in patients with unexplained infertility. Fertil Steril. 2003;79:74-80.

3. Mackenna A, Barratt CL, Kessopoulou E, Cooke I. The contribution of a hidden male factor to unexplained infertility. Fertil Steril. 1993;59:405-11.

4. Garrido N, Remohi J, Martinez-Conejero JA, Garcia-Herrero S, Pellicer A, Meseguer M. Contribution of sperm molecular features to embryo quality and assisted reproduction success. Reprod Biomed Online. 2008;17:855-65.
5. Chen X, Zhang W, Luo Y, Long X, Sun X. Predictive value of semen parameters in in vitro fertilisation pregnancy outcome. Andrologia. 2008:41:111-7.

6. Pixton KL, Deeks ED, Flesch FM, Moseley FL, Björndahl L, Ashton PR, Barratt $\mathrm{CL}$, Brewis IA. Sperm proteome mapping of a patient who experienced failed fertilization at IVF reveals altered expression of at least 20 proteins compared with fertile donors: case report. Hum Reprod. 2004;19:1438-47.

7. Frapsauce C, Pionneau C, Bouley J, Delarouziere V, Berthaut I, Ravel C, Antoine JM, Soubrier F, Mandelbaum J. Proteomic identification of target proteins in normal but nonfertilizing sperm. Fertil Steril. 2014;102:372-80.

8. Légaré C, Droit A, Fournier F, Bourassa S, Force A, Cloutier F, Tremblay R, Sullivan R. Investigation of male infertility using quantitative comparative proteomics. J Proteome Res. 2014;13:5403-14.

9. Cooper TG, Noonan E, von Eckardstein S, Auger J, Baker HW, Behre HM, Haugen TB, Kruger T, Wang C, Mbizvo MT, et al. World Health Organization reference values for human semen characteristics. Hum Reprod Update. 2010;16:231-45.

10. Zhou BF. Predictive values of body mass index and waist circumference to risk factors of related diseases in Chinese adult population. Zhonghua Liu Xing Bing Xue Za Zhi. 2002;23:5-10.

11. Zhou B. Coorperative meta-analysis group of working group on obesity in China. Prospective study for cut-off points of body mass indexin Chinese adults. Zhonghua Liu Xing Bing Xue Za Zhi. 2002;23:431-4.

12. Zhou BF. Predictive values of body mass index and waist circumference for risk factors of certain related diseases in Chinese adults-study on optimal cut-off points of body mass index and waist circumference in Chinese adults. Biomed Environ Sci. 2002;15:83-96.

13. Azpiazu R, Amaral A, Castillo J, Estanyol JM, Guimerà M, Ballescà JL, Balasch J, Oliva R. High-throughput sperm differential proteomics suggests that epigenetic alterations contribute to failed assisted reproduction. Hum Reprod. 2014;29:1225-37.

14. Frapsauce C, Pionneau C, Bouley J, Delarouziere V, Berthaut I, Ravel C, Antoine JM, Soubrier F, Mandelbaum J. Proteomic identification of target proteins in normal but nonfertilizing sperm. Fertil Steril. 2014;102:372-80.

15. Gotham SM, Fryer PJ, Paterson WR. The measurement of insoluble proteins using a modified Bradford assay. Anal Biochem. 1988;173:353-8.

16. Gallo JM, Escalier D, Grellier P, Precigout E, Albert M, David G, Schrével J. Characterization of a monoclonal antibody to human proacrosin and its use in acrosomal status evaluation. J Histochem Cytochem. 1991;39:273-82.

17. Chirinos M, Durand M, González-González ME, Hernández-Silva G, Maldonado-Rosas I, López P, Larrea F. Uterine flushings from women treated with levonorgestrel affect sperm functionality in vitro. Reproduction. 2017;154:607-14.

18. Gómez-Torres MJ, Medrano L, Romero A, Fernández-Colom PJ, Aizpurúa J. Effectiveness of human spermatozoa biomarkers as indicators of structural damage during cryopreservation. Cryobiology. 2017;78:90-4.

19. Sun W, Xing B, Guo L, Liu Z, Mu J, Sun L, Wei H, Zhao X, Qian X, Jiang Y, et al. Quantitative proteomics analysis of tissue interstitial fluid for identification of novel serum candidate diagnostic marker for hepatocellular carcinoma. Sci Rep. 2016;6:26499.

20. Ding C, Jiang J, Wei J, Liu W, Zhang W, Liu M, Fu T, Lu T, Song L, Ying W, et al. A fast workflow for identification and quantification of proteomes. Mol Cell Proteom. 2013;12:2370-80.

21. Xu J, Li L, Yu G, Ying W, Gao Q, Zhang W, Li X, Ding C, Jiang Y, Wei D, et al. The neddylation-cullin 2-RBX1 E3 ligase axis targets tumor suppressor RhoB for degradation in liver cancer. Mol Cell Proteom. 2015;14:499-509.

22. Mi H, Muruganujan A, Casagrande JT, Thomas PD. Large-scale gene function analysis with the PANTHER classification system. Nat Protoc. 2013;8:1551-66.

23. da Huang W, Sherman BT, Lempicki RA. Systematic and integrative analysis of large gene lists using DAVID bioinformatics resources. Nat Protoc. 2009:4:44-57.

24. Szklarczyk D, Franceschini A, Wyder S, Forslund K, Heller D, Huerta-Cepas J. Simonovic M, Roth A, Santos A, Tsafou KP, et al. STRING v10: proteinprotein interaction networks, integrated over the tree of life. Nucleic Acids Res. 2015;43:D447-52.

25. Liu J, Zhu P, Wang WT, Li N, Liu X, Shen XF, Wang YW, Li Y. TAT-peroxiredoxin 2 fusion protein supplementation improves sperm motility and 
DNA integrity in sperm samples from asthenozoospermic men. J Urol. 2016;195:706-12.

26. Tardif S, Guyonnet B, Cormier N, Cornwall GA. Alteration in the processing of the ACRBP/sp32 protein and sperm head/acrosome malformations in proprotein convertase 4 (PCSK4) null mice. Mol Hum Reprod. 2012;18(6):298-307.

27. Buffone MG, Foster JA, Gerton GL. The role of the acrosomal matrix in fertilization. Int J Dev Biol. 2008;52:511-22.

28. Tanphaichitr N, Kongmanas K, Kruevaisayawan H, Saewu A, Sugeng C, Fernandes J, Souda P, Angel JB, Faull KF, Aitken RJ, et al. Remodeling of the plasma membrane in preparation for sperm-egg recognition: roles of acrosomal proteins. Asian J Androl. 2015;17:574-82.

29. Yu Y, Xu W, Yi YJ, Sutovsky P, Oko R. The extracellular protein coat of the inner acrosomal membrane is involved in zona pellucida binding and penetration during fertilization: characterization of its most prominent polypeptide (IAM38). Dev Biol. 2006;290:32-43.

30. Yu Y, Vanhorne J, Oko R. The origin and assembly of a zona pellucida binding protein, IAM38, during spermiogenesis. Microsc Res Tech. 2009;72:558-65

31. Mori E, Kashiwabara S, Baba T, Inagaki Y, Mori T. Amino acid sequences of porcine Sp38 and proacrosin required for binding to the zona pellucida. Dev Biol. 1995;168:575-83.

32. Yatsenko AN, O'Neil DS, Roy A, Arias-Mendoza PA, Chen R, Murthy LJ, Lamb DJ, Matzuk MM. Association of mutations in the zona pellucida binding protein 1 (ZPBP1) gene with abnormal sperm head morphology in infertile men. Mol Hum Reprod. 2012;18:14-21.
33. Yoshinaga K, Toshimori K. Organization and modifications of sperm acrosomal molecules during spermatogenesis and epididymal maturation. Microsc Res Tech. 2003;61:39-45.

34. Buffone MG, Hirohashi N, Gerton GL. Unresolved questions concerning mammalian sperm acrosomal exocytosis. Biol Reprod. 2014;90:112.

35. Bedford JM. Site of the mammalian sperm physiological acrosome reaction. Proc Natl Acad Sci USA. 2011:108:4703-4.

36. Green S, Fishel S, Rowe P. The incidence of spontaneous acrosome reaction in homogeneous populations of hyperactivated human spermatozoa. Hum Reprod. 1999;14:1819-22.

37. Samavat J, Natali I, Degl'Innocenti S, Filimberti E, Cantini G, Di Franco A, Danza G, Seghieri G, Lucchese M, Baldi E, et al. Acrosome reaction is impaired in spermatozoa of obese men: a preliminary study. Fertil Steril. 2014;102(1274-1281):e2.

38. Du Plessis SS, Cabler S, McAlister DA, Sabanegh E, Agarwal A. The effect of obesity on sperm disorders and male infertility. Nat Rev Urol. 2010;7:153-61.

39. Plachot M, Mandelbaum J, Junca AM. Acrosome reaction of human sperm used for in vitro fertilization. Fertil Steril. 1984;42:418-23.

40. Wiser A, Sachar S, Ghetler Y, Shulman A, Breitbart H. Assessment of sperm hyperactivated motility and acrosome reaction can discriminate the use of spermatozoa for conventional in vitro fertilisation or intracytoplasmic sperm injection: preliminary results. Andrologia. 2014;46:313-5.
Ready to submit your research? Choose BMC and benefit from:

- fast, convenient online submission

- thorough peer review by experienced researchers in your field

- rapid publication on acceptance

- support for research data, including large and complex data types

- gold Open Access which fosters wider collaboration and increased citations

- maximum visibility for your research: over 100M website views per year

At BMC, research is always in progress.

Learn more biomedcentral.com/submissions 